\title{
First report of a 16SrXIV, 'Candidatus Phytoplasma cynodontis' group phytoplasma associated with coconut yellow decline in Malaysia
}

\begin{abstract}
The coconut palm (Cocos nucifera) is an important perennial oil crop cultivated throughout Malaysia and is used for its aesthetic appeal in landscapes. Symptoms of yellow decline have been observed in a plantation in Selangor, similar to those caused by phytoplasma groups of the lethal yellowing (16SrIV) type recorded from the Americas, Caribbean and Africa, and similar also to Stolbur (16SrXII) associated with Kalimantan wilt in Indonesia. Although not widespread, this is the first report on such symptoms in coconut in Malaysia. The lower canopy foliage initially turns light yellow and eventually light-brown, spreading rapidly to the younger leaves. Also severe chlorosis of the emerging spear leaf, inflorescence necrosis, premature nut fall, and gradual collapse of fronds occur, and immature palms generally die within 5 months of initial symptom appearance. Samples were collected from 20 affected and five symptomless Malayan red dwarf coconut palms. Malayan yellow dwarf and some Malayan tall coconuts exhibited similar symptoms, but were not tested in this study. Total DNA was extracted and assayed in a nested PCR with universal phytoplasma primer pairs $\mathrm{P} 1 / \mathrm{P} 7$ followed by either R16F2/R16R2 or fU5/ rU3. Products of the expected size (approximately $1200 \mathrm{bp}$ and $880 \mathrm{bp}$, respectively) were amplified from 8/20 plants with symptoms but not from any symptomless coconut palms. Amplification also failed when the LY (16SrIV) specific primers LY16Sf/LY16Sr were used for nested PCR on P1/P7 first round PCR samples (Harrison et al., 2002), as well as when LY non-ribosomal specific primers LYC24F/LYC24R (Harrison et al ., 1994) were used, indicating that the phytoplasma was not from the 16SrIV group. The phytoplasma 16SrDNA was cloned and sequenced (GenBank Acc. No. EU328159) and showed the highest homology (99\%) with those of Bermuda grass white leaf phytoplasma from Thailand (AF248961) and Malaysia (EU294011), members of the 16SrXIV 'Candidatus Phytoplasma cynodontis' group. This group has previously been found associated with a decline disease in date palm in Sudan (Cronjé et al ., 2000). However, this is the first report of a 16SrXIV phytoplasma associated with yellow decline in Malaysia.
\end{abstract}

Keyword: Candidatus Phytoplasma, Candidatus Phytoplasma cynodontis 